\title{
Age-related changes in electrophysiological and neuropsychological indices of working memory, attention control, and cognitive flexibility
}

\author{
Carrie Brumback Peltz, Gabriele Gratton and Monica Fabiani* \\ Psychology Department, Beckman Institute, University of Illinois at Urbana-Champaign, Urbana, IL, USA
}

Edited by:

Shulan Hsieh, National Cheng Kung

University, Taiwan

Reviewed by:

Francisco Barcelo, University of llles Balears, Spain

Richard Heitz, Vanderbilt University, USA

*Correspondence:

Monica Fabiani, Psychology

Department, Beckman Institute,

University of Illinois at Urbana-

Champaign, $405 \mathrm{~N}$, Mathews Avenue,

Urbana, IL 61801, USA.

e-mail: mfabiani@illinois.edu
Older adults exhibit great variability in their cognitive abilities, with some maintaining high levels of performance on executive control tasks and others showing significant deficits. Previous event-related potential (ERP) work has shown that some of these performance differences are correlated with persistence of the novelty/frontal P3 in older adults elicited by task-relevant events, presumably reflecting variability in the capacity to suppress orienting to unexpected but no longer novel events. In recent ERP work in young adults, we showed that the operation-span (OSPAN) task (a measure of attention control) is predictive of the ability of individuals to keep track of stimulus sequencing and to maintain running mental representations of task stimuli, as indexed by the parietally distributed P300 (or P3b). Both of these phenomena reflect aspects of frontal function (cognitive flexibility and attention control, respectively). To investigate these phenomena we sorted both younger and older adults into low- and high-working memory spans and low- and high-cognitive flexibility subgroups, and examined ERPs during an equal-probability choice reaction time task. For both age groups (a) participants with high OSPAN scores were better able to keep track of stimulus sequencing, as indicated by their smaller P3b to sequential changes; and (b) participants with lower cognitive flexibility had larger P3a than their high-scoring counterparts. However, these two phenomena did not interact suggesting that they manifest dissociable control mechanisms. Further, the fact that both effects are already visible in younger adults suggests that at least some of the brain mechanisms underlying individual differences in cognitive aging may already operate early in life.

Keywords: aging, working memory, operation-span task, cognitive flexibility, fluid intelligence, P300, event related brain potentials

\section{INTRODUCTION}

Older adults often complain about a reduction in their capacity to keep track of relevant information and maintain attention on specific events for extended periods of time, especially in the face of distraction. Investigators have demonstrated that these problems are associated with age-related changes in working memory and attention control (e.g., Park et al., 1996; Verhaeghen and Cerella, 2002; Fabiani and Gratton, in press). Among the theories proposed to account for these age-related cognitive declines are those suggesting that working memory function is particularly affected by aging (Craik et al., 1990), as well as those arguing that with aging it becomes more difficult to suppress the processing of distracting or novel stimuli (Hasher and Zacks, 1988; see also Hasher et al., 2008).

These theories are unified by the idea that working memory can be construed as a function primarily dependent on attention mechanisms: working memory is made up of the mental representations that are kept within the focus of attention while excluding task-irrelevant information (Kane and Engle, 2000; Hasher et al., 2008; see also Cowan, 1995). Thus, it is possible to hypothesize that deficits in working memory, such as those observed in aging, may in fact be associated with deficits in the ability to focus attention on task-relevant items and avoid the effects of distraction. Indeed,
Engle (2002) has argued that most individual differences observed in working memory capacity can be accounted for by the ability of individuals to maintain attentional focus. As a consequence, he and his colleagues developed a test of working memory function, called operation-span (or OSPAN), which is designed to assess the number of words that individuals can remember for a short period of time in the presence of a distracting task (simple mathematical operations). They went on to show that OSPAN scores predict individual differences in a variety of tasks, ranging from vocabulary learning to contract bridge playing (Engle, 2001). Although most of the research using OSPAN has been conducted on younger adults, some work on older adults has shown a decline in OSPAN scores with age, supporting the claim that the ability to maintain information in working memory in the presence of interference is in fact sensitive to age (Rhodes and Kelley, 2005; however see also Hambrick and Engle, 2002). In addition, Engle et al. (1999) have found that fluid intelligence, which is characterized by the capacity of quickly abstracting rules in a flexible manner, is moderately $(r \approx 0.4)$ correlated with OSPAN, at least in young adults. In turn, fluid intelligence is known to decline in normal aging compared to crystallized intelligence (Baltes et al., 1999). This suggests that working memory function may be critical for fluid intelligence (e.g., Carpenter et al., 1990; Prabhakaran et al., 1997; Engle et al., 1999), 
and is best understood when working memory is considered as the ability of "working-with-memory," as was proposed by Moscovitch and Winocur (1992).

Since the time of its first report (Sutton et al., 1965; for a review see Fabiani et al., 2007), the P300 component of the event-related brain potential (ERP) has also been linked to both attention and working memory. In fact a P300 is elicited only when stimuli need to be attended (Sutton et al., 1967), whereas its amplitude is modulated by stimulus probability (Duncan Johnson and Donchin, 1977) and by variations in the stimulus sequence (Squires et al., 1976). One of the most influential theories of P300 (Donchin, 1981; see also Donchin and Coles, 1988a,b), interprets the enhancement in P300 amplitude in response to a change in the stimulus sequence as a manifestation of the amount of "updating" of working memory content that is required by the presentation of a particular stimulus: when a stimulus has been recently processed its representation is likely to be still present in working memory (hence little updating is needed and P300 amplitude is small). Conversely, when a stimulus is presented after a longer interval, its representation may have faded from working memory and more updating is needed (hence a large P300 is elicited). Although other interpretations of these P300 effects have been proposed (Desmedt, 1980; Rösler, 1983; Johnson, 1986; Verleger, 1988), it is clear that a discrepancy between the current item and some form of memory representation of the items immediately preceding it is a critical factor in determining P300 amplitude (e.g., Squires et al., 1976, 1977; Klein et al., 1984), providing a clear link with the activation of working memory and attention control mechanisms. In other words, this view implies that the amplitude of the P300 elicited by a stimulus can be used to estimate the extent to which its representation in working memory has decayed since its last appearance, with more faded representations requiring more "updating" and thus eliciting larger P300s.

Since the original presentation of the "context updating" model, evidence has accumulated that the parietal-maximum P300 (or P3b, to which this model refers) is not the only form of ERP activity observed in response to attended targets between 300 and $700 \mathrm{~ms}$ from stimulation. A particularly prominent activity, variably labeled P3a, novelty $\mathrm{P} 3$, or orienting $\mathrm{P} 3$, is elicited in response to novel targets early within the same time interval. In a recent review, Polich (2007) proposed that this frontal component represents the activity of a separate system, whose operation is invoked when there is a need to shift attentional or processing sets from one condition to another. Consistent with this view, Barceló and his collaborators (Barceló et al., 2002, 2006; see also Jost et al., 2008) observed that this component is elicited when a stimulus requires to switch between processing rules. According to Polich (2007), these two components, the frontal P300 and the parietal P300, represent the operation of an attention shift system (linked to dopaminergic pathways) and of an attention maintenance system (linked to noradrenergic pathways), respectively. This view is reminiscent of the distinction between the ventral (exogenous) and dorsal (endogenous) fronto-parietal attention systems proposed by Corbetta and Shulman (2002) on the basis of neuroimaging data.

As described above, OSPAN scores are supposed to reflect the ability of individuals to maintain information in working memory. In a previous study we hypothesized that OSPAN scores would therefore predict individual differences in the ability to keep track of stimulus sequences (Brumback et al., 2005). Specifically, we hypothesized that subjects with low OSPAN scores should have particularly large parietal P300s to stimulus changes within a sequence, where the eliciting stimulus is re-presented after an interval in which stimuli of a different type were presented instead. This prediction could be considered counterintuitive, at least at first glance, because low OSPAN subjects should have reduced attention abilities, and P300 has often been considered an index of the amount of attention subjects pay to particular stimuli (Becker and Shapiro, 1980; Johnson, 1986). However, if one considers working memory as a "running attention control system," subjects with low OSPAN should have a smaller (and/or less stable) focus of attention, presumably because they are easily distracted and inappropriately deploy attentional resources away from the task at hand. As a consequence, memory representations may fall out of their attention focus more quickly when not in immediate use, and may require more "updating" to be reactivated (hence the larger P300 when a change occurs in a sequence of repeated stimuli). The results confirmed our predictions: young adults with low OSPAN scores (compared to those with high OSPAN scores) did produce an enhanced parietal P300 to items that (although previously presented) differed from their immediately preceding items. They also had a reduced N100 and P150 ERP responses to target stimuli, further suggesting a reduced attention focus (Brumback et al., 2004).

Most of the findings that have led to our theoretical understanding of the parietal P300 response, as well as the Brumback et al. (2005) study, have been obtained in young adults. However, the P300 has also been extensively studied in older adults. One of the most striking findings observed in aging is a change in the scalp distribution of P300, consisting in a reduction in amplitude at parietal electrodes, and a marked increase in amplitude at frontal locations (for reviews see Friedman et al., 1997, 2001; see also Fjell and Walhovd, 2001). This change in P300 scalp distribution has been attributed to a reduced suppression of the frontally distributed and overlapping "novelty" response (see Polich, 2007). For instance, Fabiani and Friedman (1995) found that the ERPs elicited by target items that had been previously presented, but were still occurring randomly and therefore unexpectedly, differed between younger and older adults. Namely, older adults had a P300-like response characterized by a marked amount of frontal scalp activity (labeled “novelty P3," Knight, 1987), which was absent in younger adults. This response resembled that elicited by entirely novel stimuli (i.e., stimuli that had never been presented before and were unexpected within the task context). Indeed, Fabiani and Friedman (1995) showed that both younger and older adults exhibit this type of novelty/orienting response ${ }^{1}$ to the repeated but still unexpected items at first. However, this response subsides quickly with stimulus repetition in younger adults, whereas it remains large in many older adults. Furthermore, Fabiani et al. (1998) showed that those older adults exhibiting the most pronounced perseveration of this orienting response to repeated items performed more poorly on neuropsychological tests of frontal lobe function, such as the Wisconsin card sorting test (WCST). This suggests that the

${ }^{1}$ Although Fabiani and Friedman (1995) only recorded ERPs, and therefore could not record a full-fledged orienting response, frontal aspects of the P300 are often considered a component of the orienting response (Sokolov, 1963). It is in this sense that we use this term in the current study. 
persistence of this brain response in the face of stimulus repetition may reflect some form of cognitive deficit, presumably related to diminished adaptive behavior requiring inhibition (which has often been associated with frontal lobe function). This view is also supported by studies that link performance in the WCST with working memory function (Goldman-Rakic, 1987; Berman et al., 1995). Note that, according to Polich (2007), the frontal (or novelty) P300 is related to the processing that is needed when attentional sets need to be shifted. To reconcile this view with the aging results discussed above we need to assume that older adults may have particular difficulty maintaining memory sets (perhaps reflected in the diminished parietal P300) and need therefore to engage attention-shifting mechanisms more frequently than younger adults (hence the persistence of a large frontal P300).

In this sense, the lack-of-suppression of the novelty response can be considered as an example of the often-reported deficit in inhibitory processing in aging. There is ample evidence that older adults may find it difficult to suppress processing strategies that they have used with success in the recent past, but are no longer useful at present (Rabbitt, 1965; Hasher and Zacks, 1988; Hasher et al., 2008). For example, older adults often experience difficulties in inhibiting prepotent responses (e.g., Spieler et al., 1996), in rapidly switching between tasks (e.g., Kray et al., 2002; Gratton et al., 2009), and in discarding categorization rules that are no longer useful, such as those used to solve the WCST (e.g., Fabiani and Friedman, 1995; Fabiani et al., 1998). Further, there is also evidence from electrophysiological and brain imaging data that older adults may produce increased brain activity to repeated and/or irrelevant stimuli compared to younger adults (e.g., Fabiani and Friedman, 1995; Cabeza, 2002; Gazzaley et al., 2005; Fabiani et al., 2006). Although some of this additional brain activity may be construed as compensatory (for a review see Cabeza et al., 2002; but see also Schneider-Garces et al., 2010), in some cases it may be more easily interpreted as a sign of the inability to suppress or inhibit inappropriate processing (Colcombe et al., 2005; Erickson et al., 2007).

There are logical similarities between the findings summarized above: the relationship between OSPAN scores and the parietal P300's sequential effects, the one between deficits in frontal lobe function and perseveration of the orienting/frontal P300 response, and age-related changes in fluid intelligence ${ }^{2}$ may all stem from agerelated changes to the working memory system and therefore may be relevant to cognitive deficits in aging and their variability across individuals. However, the working memory and attention systems are multifaceted and thus it is also possible that these effects are separable and related to each other in a more complex manner (e.g., Corbetta and Shulman, 2002; see also Band et al., 2002). Therefore in the current study we addressed the following questions:

a. To determine whether the relationship between individual differences in working memory function and the parietal P300 sequential effects reported in Brumback et al. (2005) in younger adults could be extended to older adults.

${ }^{2}$ We consider fluid intelligence as a concept related to cognitive or mental flexibility (Colzato et al., 2006). Mental flexibility can be defined as the ability to direct one's attention to novel events, thoughts or actions, as well as the "ability to change one's mental set to find new solutions for old problems” (Barceló et al. 2002, p.1888). b. To assess whether the relationship between individual differences in measures of working memory and mental flexibility and the frontally distributed P300 observed in older adults could be extended to younger adults.

c. To determine whether these two phenomena are correlated (as both represent a consequence of individual differences in attention control abilities) or independent (perhaps mediated by different forms of attention control, or different brain mechanisms altogether).

\section{MATERIALS AND METHODS \\ SUBJECTS}

Twenty younger (ages 20-28; 10 females) and 40 older adults (ages $65-81 ; 26$ females) were recruited from the University of Illinois at Urbana-Champaign and the surrounding community for participation in a large study under which the current data were collected ${ }^{3}$. All subjects received \$15 per hour for their participation. Only data from subjects who completed the OSPAN task were used in the present study. In addition, the data from two older adults with OSPAN scores were discarded during processing due to excessively noisy ERP data. As a result, the current study included 20 younger adults (ages 20-28; 10 females) and 38 older adults (ages 65-81; 21 females). The procedures used in this study were approved by the Institutional Review Board of the University of Illinois; all participants signed informed consent.

\section{SCREENING PROCEDURES}

All participants were extensively screened. Subjects from which data were collected reported themselves as healthy and had no previous history of head trauma, were not on medications known to affect the central nervous system, had normal or corrected to normal vision, were fluent English speakers, and were right-handed. In addition, all subjects were screened for depression with the beck depression inventory (BDI, Beck et al., 1996) and dementia with the modified minimental status exam (mMMS; Mayeux, et al., 1981). All participants admitted into the study scored in the normal range on these tests.

\section{Neuropsychological tests}

All subjects were given a battery of neuropsychological tests designed to examine their cognitive functions, including the OSPAN task. In this task subjects were required to remember words while solving simple arithmetic problems. Math problems, each followed by one word, were presented sequentially (in sets of 2-6, each set-size presented three times in a pseudorandom order). At the end of each set the subject was asked to recall the words from the previous set in the order presented. The OSPAN score is the sum of the correctly recalled sets of words (possible OSPAN score range: 0-60).

Subjects were also administered several neuropsychological tests of frontal function: the WCST (Heaton, 1981), the CFL portion of the controlled word association test (Benton and Hamsher, 1976), and the alternate category Test (Benton and Hamsher, 1976). The digit copying test (Kendrick and Watts, 1999), the WAIS digitsymbol copying test, the timed box completion, and the 3, 6, and 9 letter comparison tests (Salthouse, 1992) assessed processing speed

${ }^{3}$ The unequal group sizes in the current study were determined by constraints in the recruiting for the overall project. 
and visuo-motor integration. Fluid intelligence was measured by the short version of the Raven's progressive matrices (RPM; Raven et al., 1977; Engle et al., 1999).

\section{PROCEDURES}

Event-related potentials were recorded during a visual choice reaction time (RT) task in which subjects viewed a series of X's and O's presented on a computer screen individually and in a random (Bernoulli) sequence with equal-probability (0.50/0.50). Subjects were asked to indicate which letter ( $\mathrm{X}$ or $\mathrm{O}$ ) was presented by pressing one of two response box buttons with their right or left hand. Accuracy and speed were emphasized equally. The hand to letter assignment was counterbalanced across subjects.

The subject sat $60 \mathrm{~cm}$ away from the computer screen in a soundproof experiment room adjacent to a control room. A two-way intercom system allowed communication between the two rooms. Accuracy and RT data were recorded via the response box, which was connected to the data collection computer located in the control room.

\section{Stimuli}

The X's and O's were presented in white typeface centered on a black computer screen and subtended $0.96^{\circ}$ of visual angle vertically and $0.48^{\circ}$ of visual angle horizontally. Five blocks of 100 stimuli were presented, each for $200 \mathrm{~ms}$ with a 1600 -ms inter-stimulus interval. Rest periods were given between the blocks as needed.

\section{ERP recording and analysis}

Event-related brain potentials were recorded from eight individual gold electrodes ${ }^{4}$ (Fz, Cz, Pz, T5, T6, P3, P4, and right mastoid). An electrode placed on the left mastoid was used as an on-line reference; an averaged mastoid reference computed off-line was used for analysis. The vertical and horizontal electrooculogram was recorded for off-line eye movement correction (Gratton et al., 1983). The data were bandpass-filtered $(0.01-30 \mathrm{~Hz})$ during recording and digitized at $100 \mathrm{~Hz}$ for $1600 \mathrm{~ms}$, with a 200-ms baseline.

Trials exceeding the $\mathrm{A} / \mathrm{D}$ converter range and those with activity exceeding $300 \mu \mathrm{V}$ were excluded from the analysis. Data were averaged for each subject, trial type $\mathrm{e}^{5}$, and electrode. After visually inspecting the group and individual subject average waveforms, mean amplitude measures were taken on the average waveforms within a specified time window (300-700 ms post-stimulus for both younger and older adults).

To evaluate changes in scalp distribution, we used an approach developed by Gratton et al. (1989; vector filter). This approach treats ERP data obtained at different electrodes (in this case Fz, $\mathrm{Cz}$, and $\mathrm{Pz}$ ) as a set of variates, describing a multivariate space. An important step in the procedure is the use of a "polar" notation to describe the values observed at a variety of electrodes at a particular point in time. In this fashion, amplitude and scalp distribution are distinguished from each other: amplitude is the distance from the origin (value of 0 at all electrodes), whereas scalp distribution is

\footnotetext{
${ }^{4}$ The small number of electrodes was necessitated by the recording of the eventrelated optical signal, which was required for the larger study under which these data were collected (different tasks, same recording session).

${ }^{5}$ Trial type was defined depending on the preceding trials. Given a current stimulus $\mathrm{X}$, they could be $X X X$ (same 1-back and 2-back), OOX (different 1-back and 2-back), $X O X$ (different 1-back, same 2-back), and $O X X$, (same 1-back, different 2-back).
}

described by the angles of the point ("vector") describing the scalp distribution at different electrodes with a set of axes (the electrodes themselves). This separation of amplitude and scalp distribution is particularly useful, as it addresses issues of scalp distribution interpretation reported by a number of investigators (e.g., McCarthy and Wood, 1985; Urbach and Kutas, 2002). Specifically, to describe scalp distribution, we used two values: an angle describing the ratio between the mean value across electrodes and its standard deviation (equivalent to the coefficient of variation), and an angle describing the relative contribution of central vs. frontal vs. parietal activity. For the purpose of this presentation, we label the first angle "electrode similarity" and the second "frontality." To produce more stable results, the four types of trials were combined in this analysis.

\section{RESULTS}

\section{Neuropsychological tests}

The results of the neuropsychological tests for each age group are shown in Tables 1 and 2. One of the scales with the greatest statistical separation (i.e., the largest $t$-score) between younger and older subjects was the RPM. Further, RPM was significantly correlated with other measures of frontal lobe function, such as several scales of the WCST and alternate categories, as well as with scales that have a spatial/pattern recognition component, such as boxes, digit symbols, letter completions, all with $|r(56)|>0.40, p<0.05$. Interestingly, RPM was also correlated with OSPAN $[r(56)=0.43$, $p<0.01]$ when the two age groups were combined. As in previous studies conducted in younger adults (Engle et al., 1999), RPM showed a similar correlation in the younger subjects $[r(18)=0.44$, $p<0.05$ ]; however this correlation dropped to near 0 [actually $r(36)=-0.09$, n.s.] when only the older adult group was considered.

In order to evaluate the relationship between individual differences in working memory and measures of fluid intelligence and how they influence the brain activity observed during the RT task, we used the OSPAN and RPM scales (which had the greatest inter- and intra-group spread) to stratify our sample into high and low working memory and fluid intelligence groups (see Brumback et al., 2004). For each of the two scales we conducted median splits (separately for younger and older subjects). The OSPAN criteria used for the median splits were 18.5 for the younger subjects and 11.5 for the older subjects; the RPM criteria were 11.5 for the younger and 5.5 for the older subjects. This yielded 8 groups of subjects ( 2 age groups $\times 2$ working memory groups $\times 2$ fluid intelligence groups). The number of subjects belonging to each of the eight cells of this three-way binary classification is shown in Table 1. Chi-square tests showed that the number of subjects in each cells did not vary significantly from each other [for the younger adults, chisquare $(1)=0.89$, n.s.; for the older adults, chi-square $(1)=0.11$, n.s.; across ages, $\operatorname{chi-square}(1)=0.68$, n.s.]. Average values for demographic (gender and years of education) and neuropsychological scales for each of these cells are also presented in Table 1. The high and low-RPM groups tended to differ as a function of education and gender: both of these effects were only evident in the older group, suggesting that they may be the result of life-long conditions. Note that since the OSPAN and RPM groups were sorted within each age group, this procedure amounts to a stratification of the sample, with the effects of age being largely partialed out from the effects of other groupings. 
Table 1 | Demographic and neuropsychological data for each group in the study: mean (SD).

Younger adults

\begin{tabular}{|c|c|c|c|c|c|c|c|c|}
\hline \multirow[t]{2}{*}{ Scale } & \multicolumn{2}{|c|}{ Low OSPAN } & \multicolumn{2}{|c|}{ High OSPAN } & \multicolumn{2}{|c|}{ Low OSPAN } & \multicolumn{2}{|c|}{ High OSPAN } \\
\hline & Low RPM & High RPM & Low RPM & High RPM & Low RPM & High RPM & Low RPM & High RPM \\
\hline Age & $22.7(2.3)$ & $21.3(0.6)$ & $23.8(3.2)$ & $21.8(1.0)$ & 71.1 (5.3) & $72.8(5.3)$ & $71.3(4.1)$ & $71.0(5.5)$ \\
\hline F/M & $3 / 3$ & $1 / 2$ & $3 / 2$ & $3 / 3$ & $7 / 3$ & $3 / 5$ & $7 / 3$ & $4 / 6$ \\
\hline Educ. & $16.8(1.5)$ & $15.5(0.5)$ & $17.1(2.3)$ & $16.2(0.9)$ & $15.0(2.9)$ & $17.6(2.6)$ & $16.2(2.9)$ & $18.1(2.0)$ \\
\hline Boxes & $52.2(14.0)$ & $65.3(10.8)$ & $46.2(10.0)$ & $51.2(5.3)$ & $35.2(10.6)$ & $48.6(10.7)$ & $45.1(12.9)$ & 43.7 (10.9) \\
\hline WCST & $13.7(7.6)$ & $12.3(10.1)$ & $11.2(4.6)$ & $11.7(2.9)$ & $38.3(14.0)$ & $37.3(16.8)$ & $23.3(11.2)$ & $22.4(17.1)$ \\
\hline DigCopy & 79.7 (4.8) & $81.0(8.9)$ & $77.6(8.4)$ & $80.0(3.9)$ & $57.8(11.0)$ & $68.5(14.8)$ & $68.7(16.0)$ & 70.4 (11.0) \\
\hline DigSymb & $45.7(2.5)$ & $52.0(2.0)$ & $47.6(6.4)$ & $52.8(5.2)$ & $30.0(2.8)$ & $34.4(5.3)$ & $37.1(6.6)$ & $37.7(5.6)$ \\
\hline LetComp & $22.0(2.4)$ & $24.0(1.0)$ & $22.8(3.5)$ & $25.5(2.0)$ & $13.2(2.0)$ & $15.6(2.1)$ & $17.0(2.7)$ & $17.2(1.6)$ \\
\hline
\end{tabular}

WCST, total number of errors.

Table 2 | ANOVA on demographic and neuropsychological data - df (1, $50)$; only significant $F$ s are shown.

\begin{tabular}{|c|c|c|c|c|c|c|c|}
\hline Factors & Age & OSPAN & RPM & $A \times 0$ & $A \times R$ & $O \times R$ & $A \times O \times R$ \\
\hline \multicolumn{8}{|l|}{ Gender } \\
\hline Educ. & & & & & $6.49 *$ & & \\
\hline mMMS & $4.93^{*}$ & & & & & & \\
\hline Boxes & $11.18^{* * *}$ & & $5.70 *$ & $3.95^{\dagger}$ & & & \\
\hline WCST & $14.50^{* * *}$ & $3.01+$ & & & & & \\
\hline DigCopy & $16.24 * * *$ & & & & & & \\
\hline DigSymb & $104.82^{* * *}$ & $5.25^{*}$ & $7.11^{*}$ & & & & \\
\hline LetComp & $108.37^{* * *}$ & $6.53^{*}$ & $5.94 *$ & & & & \\
\hline AltCateg & $16.51 * * *$ & & & & & & \\
\hline OSPAN & $16.82^{* * *}$ & & & & & & \\
\hline RPM & $62.46 * * *$ & & & & & & \\
\hline
\end{tabular}

${ }^{*}{ }^{*} p<0.001 ;{ }^{*} p<0.01 ;{ }^{*} p<0.05 ;{ }^{+} p<0.10$.

$A$, age; $O, S P A N ; R, R P M$ (Raven progressive matrices).

\section{Behavioral data}

For the analysis of RTs (see Table 3), trials were sorted as a function of the relationship (same or different) with the immediately previous trial (1-back) and the trial two back (2-back). This yielded four categories of trials. Sequential trees were computed by averaging across all trials, and comparing trials differing (or not) from the preceding trial, and those differing (or not) from the trial 2-back. This was done separately for each subject. The average sequential trees across subjects, sorted on the basis of age, OSPAN group, and RPM group are presented in Figure 1 (top). A mixed design ANOVA with three between-subject factors (age, OSPAN, and RPM) and two within-subject factors (1-back and 2-back) was run to evaluate the statistical significance of the effects. This ANOVA revealed a main effect of age

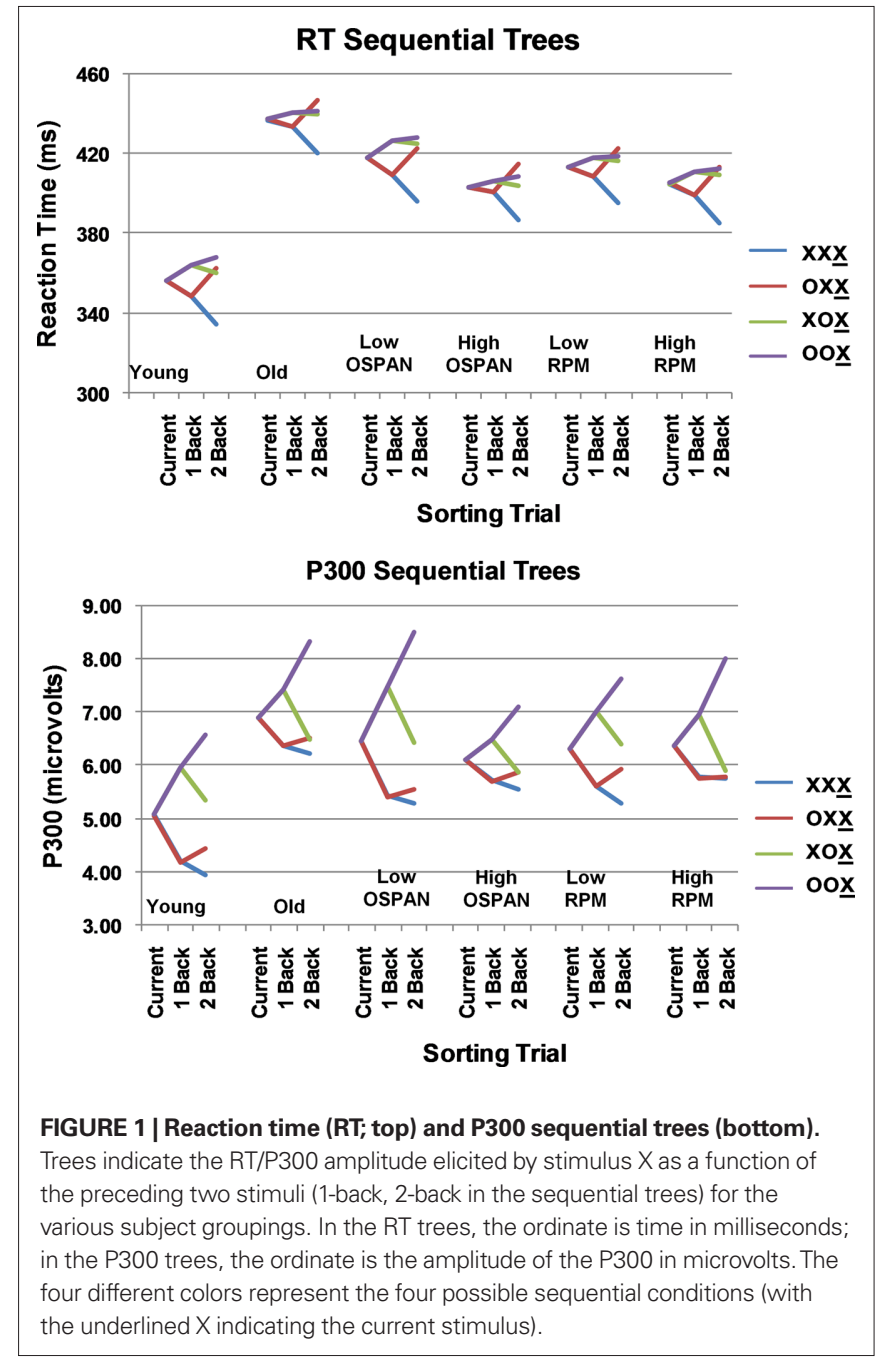


$[F(1,50)=44.35, p<0.001]$, with the younger adults being faster than the older adults. There were significant effects of sequential level: for 1-back $[F(1,50)=12.28, p<0.001]$ and for 2-back $[F(1,50)=50.13, p<0.001]$. There was also a significant interaction between 1-back and 2-back $[F(1,50)=30.59, p<0.001]$. Inspection of Figure 1 (top) indicates that the sequential effects on RT are entirely attributable to faster responses for the condition in which the current item was identical to the previous two items (XXX), whereas having only one item identical to the previous one (OXX) does not affect RT compared to the alternation condition (XOX). There were no significant interactions between age and sequential level, indicating that younger and older adults were equally influenced by stimulus sequence. The only other effect of relevance was a marginally significant twoway interaction between 1-back and OSPAN group, showing a more pronounced RT sequential effect for the low OSPAN group (indicated by the difference between the red and purple lines in Figure 1 top for this group), $F(1,50)=2.88, p<0.10$ (note that this would be significant in a directional test, which would be appropriate here). Finally, we also examined whether RT was influenced by OSPAN group irrespective of sequential level (i.e., considering all trials). For the older adults only there was a significant negative correlation between OSPAN score and overall mean RT, $r=-0.30, p<0.05$, one-tailed. The corresponding correlation for the younger adults was not significant $(r=-0.20)$. RPM grouping did not have any significant effects or interactions for the sequential tree and overall RT analyses.

Accuracy was over $90 \%$ for all groups and conditions. Because of these high values, we applied a logit transform before submitting the data to statistical analysis, and collapsed the data across sequential levels. A between-subject design ANOVA including age, OSPAN, and RPM as factors revealed only a marginal interaction between age and OSPAN $[F(1,50)=-2.87, p<0.10]$. This interaction was due to a reduced accuracy for the older adults with low OSPAN $(M=0.944)$, compared to their high OSPAN counterparts $(M=0.964)$. This difference was amplified when assessed as a correlation between the actual OSPAN score (instead of the group assignment) and accuracy $(r=0.33$, $p<0.025$ one-tailed). A smaller difference in the same direction was observed in the younger adults $(M=0.957$ and 0.966 , respectively).

Overall, these behavioral data indicate that (a) the older adults had overall lower performance than younger adults; (b) subjects RTs were influenced by the stimulus sequence irrespective of age; and (c) both of these effects were accentuated in subjects with low OSPAN scores.

Table 3 | Mean reaction time and accuracy scores (with SD in parentheses) for the two age groups and the two OSPAN groups.

\begin{tabular}{llllll}
\hline & \multicolumn{2}{c}{ Younger adults M(SD) } & & \multicolumn{2}{c}{ Older adults M(SD) } \\
\cline { 2 - 3 } & High OSPAN & Low OSPAN & & High OSPAN & Low OSPAN \\
\hline Reaction & 355.27 & 360.06 & & 431.73 & 444.58 \\
time (ms) & $(32.55)$ & $(40.18)$ & & $(40.21)$ & $(51.28)$ \\
Accuracy & 0.95 & 0.96 & & 0.96 & 0.93
\end{tabular}

\section{Event-related potential data}

The ERPs were analyzed using the same trial-sorting procedure used for RTs. Grand averages were computed for each age group, and for subjects sorted on the basis of OSPAN and/or RPM scores. Grand average waveforms from the three midline electrodes ( $\mathrm{Fz}, \mathrm{Cz}$, and $\mathrm{Pz}$ ) for younger and older adults are presented in Figure 2 for OSPAN sorting and in Figure 3 for RPM sorting (both median splits were conducted separately in younger and older subjects, as in the previous analyses). The waveforms presented in these Figures depict the brain responses when the current stimulus was identical to the two immediately preceding stimuli (XXX condition, where $\mathrm{X}$ is the current stimulus) or different from both preceding stimuli (OOX condition).

The most prominent brain response observed in these figures is a positivity, with a latency between 300 and $700 \mathrm{~ms}$, largest at the parietal electrode, and generally larger for trials differing from the preceding ones, which we identify as the parietal P300 component of the ERP.

The grouping variables (age, OSPAN scores, and RPM scores) appear all to influence the P300 complex, though in different ways. Age appears to be correlated with a change in scalp distribution, so that a smaller difference is observed between frontal and parietal locations for older adults than for younger adults. This finding is

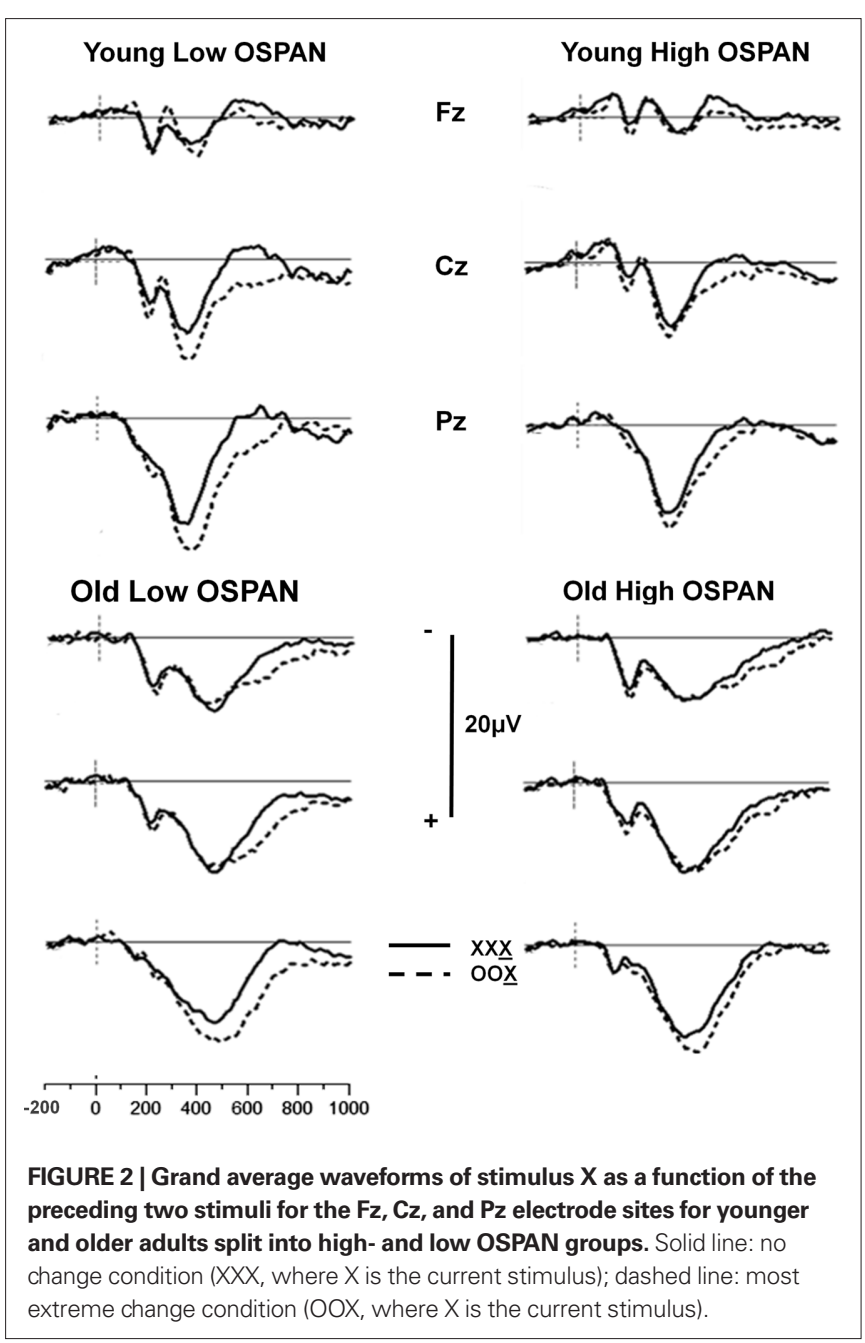




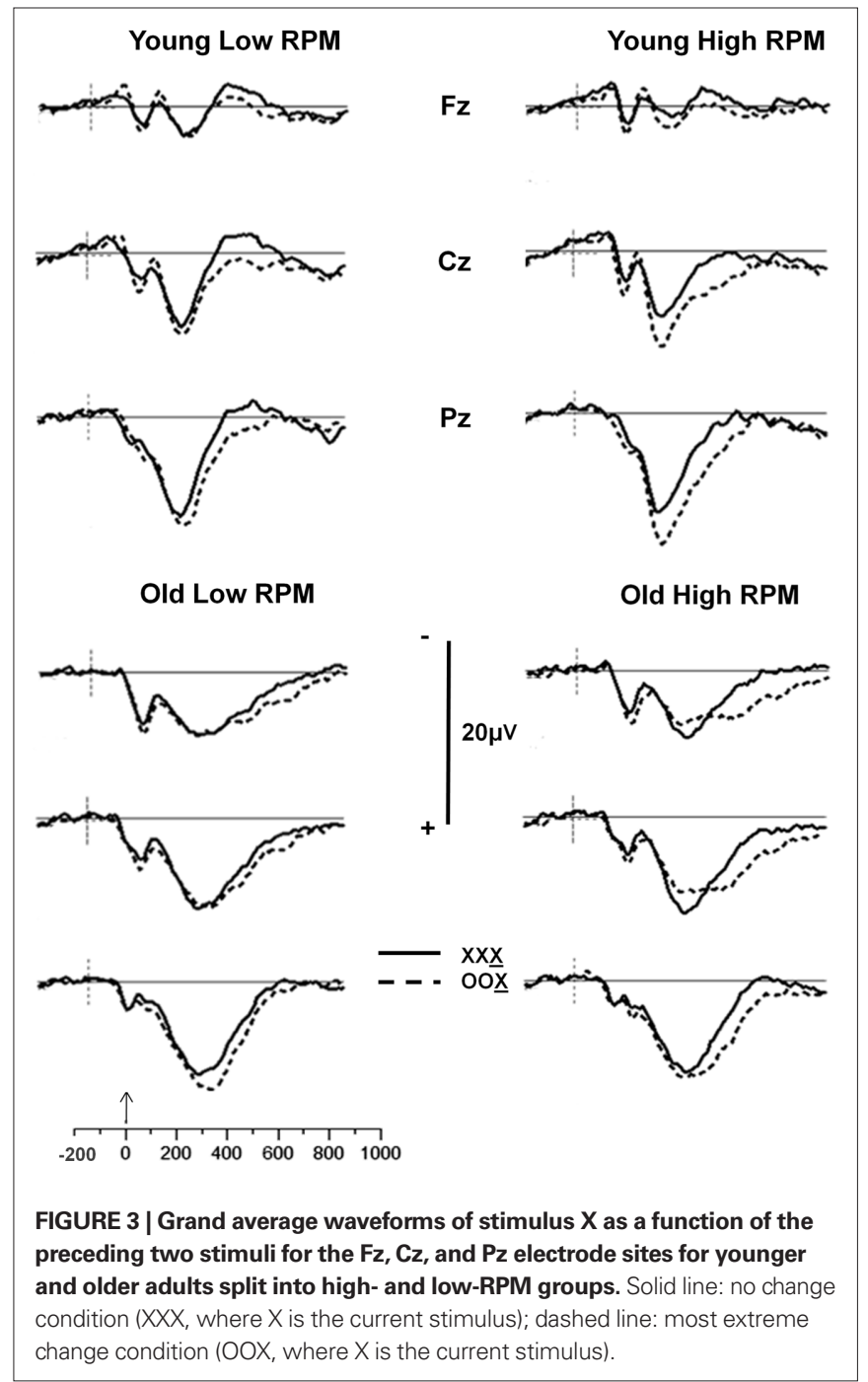

consistent with previous reports (e.g., Fabiani and Friedman, 1995; for reviews see Friedman et al., 1997, 2001), and could be attributed to a larger frontal P3 (or P3a) in the older adults. RPM grouping also appears to be related to differences in P300 scalp distribution, with a smaller parietal, and a more pronounced central and frontal activity in the low compared to the high RPM groups, regardless of age. Thus, RPM grouping seems to produce effects that mirror those of aging.

Operation-span grouping appears mostly to predict the amplitude of the sequential effects (i.e., the difference between mismatching and matching trials). This is particularly evident when the waveforms from the matching condition (XXX) are subtracted from the mismatching condition (OOX). These waveforms (from the Pz electrode) are shown in Figure 4 (top). Similar waveforms computed for RPM groupings (Figure 4, bottom) show smaller and inconsistent effects.

To evaluate these visual impressions, we quantified the amplitude of parietal P300 by computing the average amplitude of the activity (as described in the Materials and Methods) for each subject, trial type, and midline electrode. To evaluate sequential effects, the results were averaged across electrode locations, and submitted to an ANOVA design identical to that used for RTs. The P300 amplitude sequential trees are presented in Figure $\mathbf{1}$ (bottom). The results of the ANOVA indicated clear effects of stimulus sequence on P300 amplitude: the 1-back effect yielded an $F(1,50)=21.79$, $p<0.001$, the 2-back effect an $F(1,50)=30.23, p<0.001$, and the 1-back $\times 2$-back interaction was also significant $[F(1,50)=7.94$, $p<0.01]^{6}$. The $\mathrm{P} 300$ was always larger to mismatching trials, but the effect was particularly large for the "double mismatch" (OOX) condition. Of the grouping variables, only OSPAN interacted with the sequential effects: the OSPAN $\times 1$-BACK interaction was significant $[F(1,50)=21.79, p<0.05]$, with low OSPAN subjects showing a greater sequential effect than high OSPAN subjects, irrespective of age (as clearly visible in Figure 4). This finding replicates the results of Brumback et al. (2005) and extends them to the older adult group.

In order to assess whether the frontal P300 was also sensitive to sequential effects we repeated this ANOVA on the measurements from the frontal electrode alone. Although the frontal P300 was larger for older than for younger adults [main effect of age, $[F(1$, $50)=13.58, p<0.001]$, there were no significant sequential effects or interactions.

As described in the Section "Materials and Methods," the vector filter approach (Gratton et al., 1989) was used to analyze changes in scalp distribution. We used two values to describe scalp distribution: an angle describing the ratio between the mean value across electrodes and its standard deviation (labeled "electrode similarity"), and an angle describing the relative contribution of frontal vs. central vs. parietal activity (labeled "frontality"). To produce more stable results, the four types of trials were combined in this analysis. Two separate ANOVAs were run, one for each of the two measures, each with three between-subjects factors (age, OSPAN group, and RPM group). The ANOVA on "electrode similarity" yielded a significant effect of age group, $F(1,50)=5.20, p<0.05$, but no other significant main effect or interaction. As expected, the similarity between electrodes was greater for older than for younger adults (see Figure 5, top right). The ANOVA on "electrode orientation" yielded a significant effect of RPM group, $F(1$, $50)=6.90, p<0.05$, indicating a relative greater contribution of central and frontal electrodes, and a relatively smaller parietal activity for the low-RPM group compared to the high RPM group. No other significant main effect or interaction emerged (see Figure 5, bottom left). These results indicate that, regardless of age, a lowRPM score may be associated with a relatively greater use of brain structures leading to a fronto-central scalp distribution of P300, rather than a parietal-maximum scalp distribution (typical of high RPM subjects), which in turn may indicate an elicitation of novelty/ orienting processes that are not required by the high RPM subjects. Interestingly, this is not the case when participants are separated on the basis of their OSPAN score (also shown in Figure 5).

\section{DISCUSSION}

In our previous work we had identified two types of ERP phenomena that can be recorded during relatively simple classification tasks, and that appear to be connected to working memory function and aging. The first is the presence of a frontally dis-

${ }^{6}$ To ensure that these effects were not due to just a few subjects with large P300 amplitudes we repeated the statistical analysis on normalized data. This analysis yielded virtually identical results. 


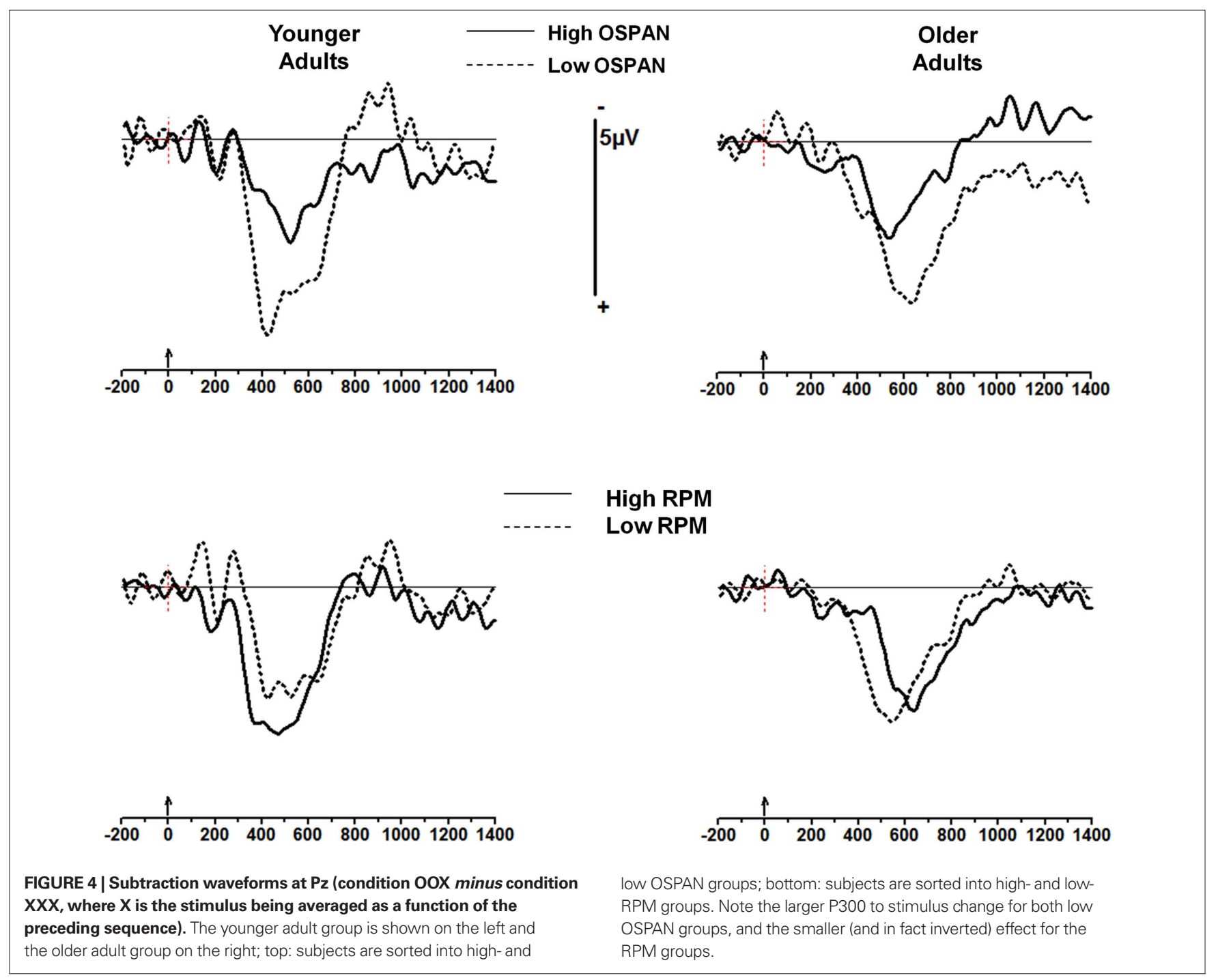

tributed scalp potential, the novelty $\mathrm{P} 3$ or $\mathrm{P} 3 \mathrm{a}$, typically obtained in younger adults when they are processing novel information, and in older adults not only under the same conditions, but even when processing information that is unexpected, but no longer novel (Fabiani and Friedman, 1995; Fabiani et al., 1998). The occurrence of this response in older adults is associated with impaired frontal lobe function, as assessed by neuropsychological tests (Fabiani et al., 1998). The second phenomenon is an exaggerated parietal P300 (or P3b) response to changes in stimulus sequences, which is shown by younger subjects with low scores in loaded tests of working memory capacity such as OSPAN (Brumback et al., 2005). This exaggerated response can be considered to be the result of a failure to maintain an appropriate working memory representation of items that had been previously presented but were superseded by more recent events. The first two questions addressed by this study were (a) whether the relationship between individual differences in working memory function and sequential effects in the parietal P300 reported in younger adults could be extended to older adults; (b) whether the relationship between individual differences in measures of working memory and mental flexibility and the persistence of a frontal P300 observed in older adults could be extended to younger adults. Both questions had a positive answer. Our results showed that the relationship between OSPAN score and sequential effects in the parietal P300 amplitude was evident in both younger and older adults, thus replicating and extending previous findings by Brumback and colleagues. Similarly, there was an inverse relationship between RPM score and frontality of $\mathrm{P} 3$ distribution, irrespective of age.

Our analytical strategy was to use an individual difference approach, and to identify subjects with high and low scores on neuropsychological tests that could be used as proxies for underlying latent variables. Specifically, we used the OSPAN test to assess working memory capacity and the RPM as a measure of fluid intelligence and cognitive flexibility. Our choice of the OSPAN test as a measure of loaded working memory capacity is supported by a number of studies that have shown that this measure is reliable and valid, and it can predict performance in a variety of different tasks (Engle, 2002). OSPAN has been used in the study of aging (e.g., Rhodes and Kelley, 2005). In our previous work, OSPAN has 


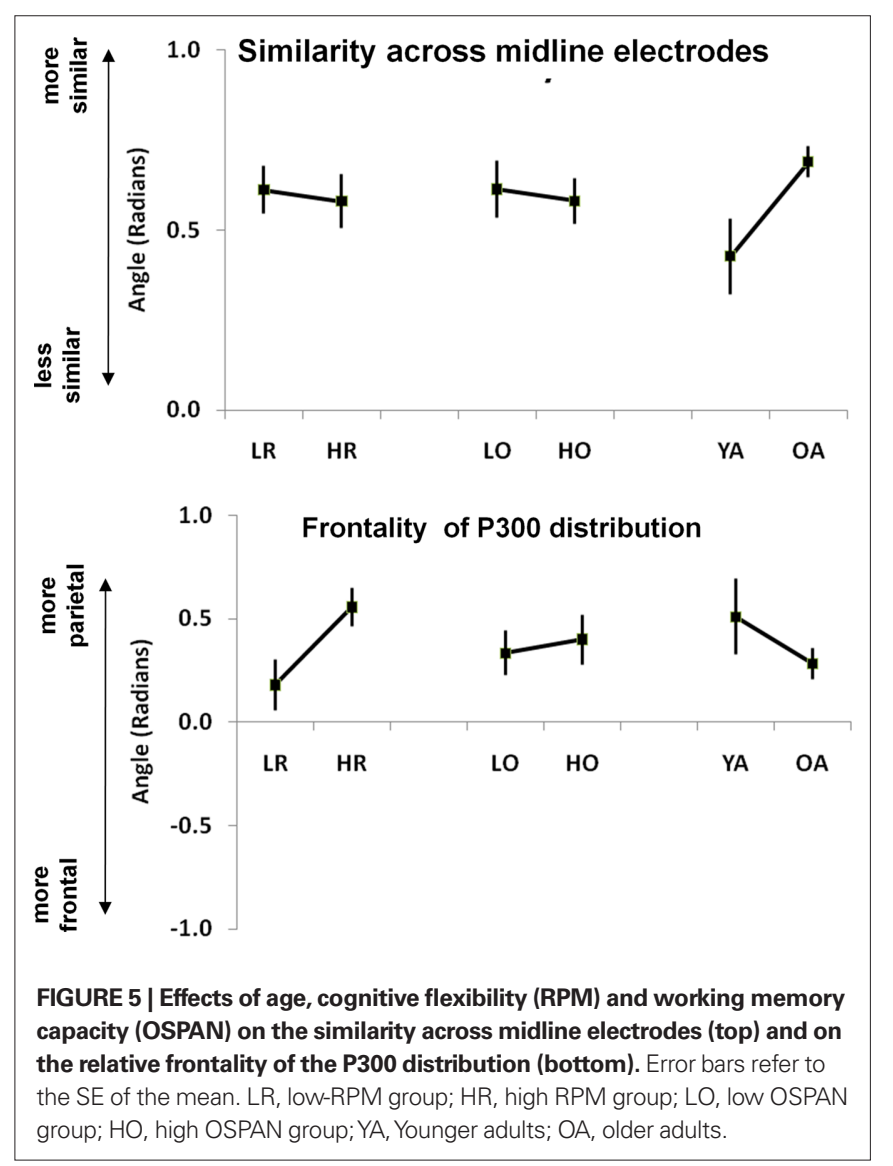

shown to be a powerful predictor of brain activity associated with the processing of unexpected but repeated items in younger adults (Brumback et al., 2004, 2005).

Performance in the RPM test has been correlated with frontal lobe function and cognitive flexibility in previous studies (e.g., Duncan et al., 1995; Colzato et al., 2006). In the current study RPM scores were significantly correlated with performance in the WCST. In addition, RPM presents certain advantages. First, it shows very consistent and large differences as a function of age. Second, it shows variability in both younger and older adults, allowing us to group subjects in both age intervals (unlike the WCST, which tends to be at ceiling in younger adults). Third, in our previous work it has shown to be a good predictor of individual differences in attention-related ERP components in younger adults (Brumback et al., 2004).

Not withstanding this supporting evidence, and as is usually the case with neuropsychological tests, RPM and OSPAN are complex measures that may reflect psychological constructs other than those we are postulating here. As such, some of the conclusions of this study need to be moderated by the possibility that other factors may underlie some of the observed effects. As mentioned earlier, OSPAN and RPM are significantly correlated in the entire group of subjects and in the younger adults but not in the older adults when they are considered separately. This relationship indicates that the two tests may share some processing abilities, at least in younger adults. Indeed, fluid intelligence has been considered to depend, at least in part, on working memory function (Carpenter et al., 1990).
However, the fact that the correlation no longer exists in older adults also suggest that the lifespan development trajectories of these two abilities are different and show dissociations that may not be evident earlier in life.

Our third and central question was whether the two P300 phenomena described above are underlined by the same factor - as, for instance, would be the case if they were both directly related to poorer attention control mechanisms. This does not appear to be the case. In fact, the patterns of effects associated with the two ERP phenomena were clearly distinct. The set of neuropsychological variables associated with each of them was not overlapping. OSPAN grouping, but not RPM grouping, was correlated with sequential effects in P300 amplitude, as well as RT and accuracy in the task (especially in older adults). RPM grouping, but not OSPAN grouping, was associated with changes in P300 scalp distribution (see Figure 5).

To evaluate the significance of these findings, it is important to consider that we used a stratified approach. Subjects were classified in eight groups using three different variables (age, OSPAN, and RPM). This approach tends to "orthogonalize" the different variables, so that it makes it easier to determine whether the effect of one variable are mediated by differences on another, or whether instead they are independent. Thus, in this case it suggests that the effects of age on the parietal P300 sequential effects may be mediated by its effect on loaded working memory span. This, in turn, strongly supports the claim that $\mathrm{P} 300$ 's sequential effects are related to the (in)ability of subjects to maintain memory representations active in the presence of interfering tasks/stimuli (which is directly assessed by the OSPAN task).

The ERP data replicate and expand our previous findings, including the observation that aging is associated with more frontal and less parietal scalp ERP activity (e.g., Fabiani and Friedman, 1995; Fabiani et al., 1998). This latter finding appears consistent with brain imaging data, suggesting increased reliance on frontal brain structures (or default network structures) by older adults compared to younger adults (Grady et al., 1998; Cabeza, 2001; Cabeza et al., 2002).

In this paper we have considered sequential effects and shifts in scalp distribution as two phenomena associated with $a$ and $b$ subcomponents of the P300. This attribution is intended here only in terms of temporal overlap. Indeed, it is likely that these two phenomena are associated with two separate ERP components, such as the $\mathrm{P} 3 \mathrm{a}$ (the frontal shift in distribution) and the P3b (the sequential effects), as described by Polich (2007; see also Dien et al., 2003). In Polich's model the P3a is associated with a frontal circuit related to novelty processing and the $\mathrm{P} 3 \mathrm{~b}$ with a temporo-parietal circuit related to holding information and attention. In this case, the first, but not necessarily the second, of these phenomena may be significantly influenced by aging - as aging leads to a more extensive scalp distribution shift, whereas the sequential effects are present as a function of OSPAN score but irrespective of age. However, in our interpretation it is not the $\mathrm{P} 3$ a response per se that is diminished in aging (or in subjects with low-RPM scores). This activity is in fact enhanced in these groups. Rather, it is the habituation process leading to the dampening of this frontal activity that is reduced in aging (see Fabiani and Friedman, 1995). Similarly, the increased sequential effects observed in subjects with low OSPAN is not a sign that these 
subjects do have a reduced $\mathrm{P} 3 \mathrm{~b}$ per se (as in fact the amplitude of $\mathrm{P} 3 \mathrm{~b}$ is enhanced), but rather a reduced ability to maintain working memory representations, leading to the enhancement.

In summary, the frontal P300 (novelty $\mathrm{P} 3$ or $\mathrm{P} 3 \mathrm{a}$ ) is not modulated by sequential trees but its amplitude reflects individual differences in cognitive flexibility (as indexed here by RPM scores), which are, in turn, highly influenced by age. This latter finding is in apparent contrast with results reported in previous studies (e.g., Barceló et al., 2002, 2006; Jost et al., 2008), who reported a large P3a after stimuli requiring a task switch in younger adults. To understand this discrepancy we should consider that the switch paradigms used by Barceló and colleagues (as well as in the Jost et al., 2008 study) are in fact quite different and more complex than the paradigm used in the current study. Most important, in those studies stimulus change included a requirement to shift attentional set. This may be an important requirement for generating a frontal (or novelty) P3 (as also suggested by Polich, 2007). In our paradigm, there are only two stimuli (each of which one-dimensional) that can be presented at any trial, and there is no need for a shift in attentional/task set. The sequential effects do not reflect such a switch, but rather the maintenance of a particular one-dimensional memory set. We submit that, for this reason, the sequential effects we observe in the current study do not involve the engagement of the frontal (or novelty) P3, but rather are sustained by the parietal P300. Interestingly, the maintenance of a particularly memory set is also required by the OSPAN task, whereas the ability to flexibly switch attentional set is probably very important for the RPM task. Thus these two sets of data do not appear as contradictory, but rather as complementary.

In this study we observed sequential effects on both the parietal P300 amplitude and RTs. This may lead to the question of whether these effects are related. However, the sequential effects on the parietal P300 and RTs were different. P300 amplitude was modulated by both 1-back and 2-back items, whereas for RT the effects were only visible for the 2-back condition. This dissociation makes it unlikely that the P300 sequential effects could be accounted for by a possible overlap of ERP activity directly related to movements, as the 1-back effects on P300 had no concurrent effect on RTs. In fact, the parietal P300 is known to be related to some advanced level of processing which, under some conditions but not others, may be related to response selection (see Kutas et al., 1977; McCarthy and Donchin, 1981; Magliero et al., 1984; Coles et al., 1985).

\section{REFERENCE}

Baltes, P. B., Staudinger, U. M., and Lindenberger, U. (1999). Lifespan psychology: theory and application to intellectual functioning. Annu. Rev. Psychol. 50, 471-507.

Band, G. P., Ridderinkhof, K. R., and Segalowitz, S. (2002). Explaining neurocognitive aging: is one factor enough? Brain Cogn. 49, 259-267.

Barceló, F., Escera, C., Corral, M. J., and Periáñez, J. A. (2006). Task switching and novelty processing activate a common neural network for cognitive control. J. Cogn. Neurosci. 18, 1734-1748.

Barceló, F., Periáñez, J. A., and Knight, R. T. (2002). Think differently: a brain orienting response to task novelty. Neuroreport 13, 1887-1892.
Beck, A. T., Steer, R. A., and Brown, G. K. (1996). Manual for the Beck Depression Inventory, 2nd Edn. San Antonio, TX: The Psychological Corporation.

Becker, D. E., and Shapiro, D. (1980). Directing attention toward stimuli affects the P300 but not the orienting response. Psychophysiology 17, 385-389.

Benton, A., and Hamsher, K. (1976). Multilingual Aphasia Exam. Iowa City, IO: University of Iowa press.

Berman, K. F., Ostrem, J. L, Randolph, C., Gold, J., Goldberg, T. E., Coppola, R., Carson, R. E., Herscovitch, P., and Weinberger,D.R.(1995). Physiological activation of a cortical network during performance of the Wisconsin Card Sorting Test: a positron emission

Although limited in terms of their spatial resolution, the data from the current study (as those from our previous ERP experiments, Fabiani and Friedman, 1995; Czernochowski et al., 2008) provide additional information by pointing out that the increased reliance on frontal activity (albeit only measured at the scalp) is variable across individuals and correlates with measures such as neuropsychological test scores or education. Many investigators are interested in determining whether frontal activity (and any additional brain activity observed in aging) is compensatory or dysfunctional (Cabeza, 2001; Park et al., 2001; Cabeza et al., 2002). Although the current study cannot answer this question, it is possible to speculate that reliance on frontal function may lead to a consumption of resources that could be otherwise dedicated to other tasks. Thus, even if this activity is compensatory for the current task, reliance on frontal function may ultimately be dysfunctional within a broader perspective, when more than one task needs to be accomplished. Of course, some benefits may also result in an indirect fashion (e.g., Sunghan et al., 2007). Further, the observation that individual differences in OSPAN and RPM scores lead to similar effects on the P300's subcomponents in younger and older adults further suggests that at least some of the changes that are typically attributed to aging may be quantitative and represent the natural progression of traits present earlier in life. In such context, they may be indicative of distributional shifts along a continuum rather than the emergence of new phenomena in aging, an idea that is also underlined in the CRUNCH model (compensation-related utilization of neural circuits hypothesis; Reuter-Lorenz and Cappell, 2008; see also Schneider-Garces et al., 2010).

\section{ACKNOWLEDGMENTS}

The work presented in this paper was supported by NIA grants \#AG21887 and 1RC1AG035927 to M. Fabiani, and by an NIMH T32 MH14257 Training Grant Fellowship to Carrie Brumback. We also thank Randy Engle for the software running the OSPAN task, Charles L. Brown III for computer programming and technical assistance on the waveform display program, and Dr. David Friedman for the use of software developed in his laboratory. Dr. Peltz is now at UC-Irvine, 843 Health Sciences Rd., 1511 Hewitt Hall, Irvine, CA 92697-1400 (cpeltz@uci.edu).

tomography study. Neuropsychologia 33, 1027-1046.

Brumback, C. R., Low, K. A., Gratton, G., and Fabiani, M. (2004). Sensory brain responses predict individual differences in working memory span and fluid intelligence. Neuroreport 15, 373-376.

Brumback, C. R., Low, K. A., Gratton, G., and Fabiani, M. (2005). Putting things into perspective: individual differences in working memory span and the integration of information. Exp. Psychol. 52, 21-30.

Cabeza, R. (2001). "Functional neuroimaging of cognitive aging," in Handbook of Functional Neuroimaging of Cognition, eds R. Cabeza and A. Kingstone (Cambridge, MA: MIT Press), 331-377.
Cabeza, R. (2002). Hemispheric asymmetry reduction in older adults: the HAROLD model. Psychol. Aging 17, 85-110.

Cabeza, R., Anderson, N. D., Loncantore, J. K., and McIntosh, A. R. (2002). Aging gracefully: compensatory brain activity in high-performing older adults. Neuroimage 17, 1394-1402.

Carpenter, P. A., Just, M. A., and Shell, P. (1990). What one intelligence test measures: a theoretical account of the processing in the Raven progressive matrices test. Psychol. Rev. 97, 404-431.

Colcombe, S. J., Kramer, A. F., Erickson, K. I., and Scalf, P. (2005). The implications of cortical recruitment and brain morphology for individual differences in 
inhibitory function in aging humans. Psychol. Aging 20, 363-375.

Coles, M. G., Gratton, G., Bashore, T. R., Eriksen, C. W., and Donchin, E. (1985). A psychophysiological investigation of the continuous flow model of human information processing. $J$. Exp. Psychol. Hum. Percept. Perform. $11,529-553$.

Colzato, L. S., van Wouwe, N. C., Lavender, T. J., and Hommel, B. (2006). Intelligence and cognitive flexibility: fluid intelligence correlates with feature "unbinding" across perception and action. Psychon. Bull. Rev. 13, 1043-1048.

Corbetta, M., and Shulman, G. L. (2002). Control of goal-directed and stimulusdriven attention in the brain. Nat. Rev. Neurosci. 3, 201-215.

Cowan, N. (1995). Attention and Memory: An Integrated Framework. New York: Oxford University Press.

Craik, F. I. M., Morris, R. G., and Gick, M. (1990). "Adult age differences in working memory," in Neuropsychological Impairments of Short-Term Memory, eds G. Vallar and T. Shallice (Cambridge: Cambridge University Press), 247-267.

Czernochowski, D., Fabiani, M., and Friedman, D. (2008). Use it or lose it? Socio-economic status mitigates age-related decline in a recency/recognition task. Neurobiol. Aging 29, 945-958.

Desmedt, J. E. (1980). "P300 in serial tasks: an essential post-decision closure mechanism," in Motivation, Motor, and Sensory Processes of the Brain. Progress in Brain Research, Vol. 54, eds H. H. Kornhuber and L. Deecke (Amsterdam: Elsevier-North Holland), 682-686.

Dien, J., Spencer, K. M., and Donchin, E. (2003). Localization of theeventrelated potential novelty response as defined by principal components analysis. Cogn. Brain Res. 17, 637-650.

Donchin, E. (1981). Surprise!...Suprise? Psychophysiology 18, 493-513.

Donchin, E., and Coles, M. G. H. (1988a). Is the $\mathrm{P} 300$ component a manifestation of context updating? Behav. Brain Sci. 11, 355372.

Donchin, E., and Coles, M. G. H. (1988b). On the conceptual foundations of cognitive psychophysiology: a reply to comments. Behav. Brain Sci. 11, 406417.

Duncan, J., Burgess, P., and Emslie, H. (1995). Fluid intelligence after frontal lobe lesions. Neuropsychologia 33, 261-268.

Duncan Johnson, C. C., and Donchin, E. (1977). On quantifying surprise: the variation of event related potentials with subjective probability. Psychophysiology 14, 456-467.
Engle, R. W. (2001). "What is working memory capacity?" in The Nature of Remembering, Essays in Honor of Robert G. Crowder, eds H. L. Roediger, J. S. Nairne, I. Neath, and A. M. Surprenant (Washington, DC: American Psychological Association Press), 297-314.

Engle, R. W. (2002). Working memory capacity as executive attention. Curr. Dir. Psychol. Sci. 11, 19-23.

Engle, R. W., Tuholski, S. W., Laughlin, J. E., and Conway, A. R. (1999). Working memory, short-term memory, and general fluid intelligence: a latentvariable approach.J. Exp. Psychol. Gen. 128, 309-331.

Erickson, K. I., Colcombe, S. J., Wadhda, R., Beherer, L., Peterson, M. S., Scalf, P. E., Kim, J. S., Alvarado, M., and Kramer,A. F. (2007). Training-induced plasticity in older adults: Effects of training on hemispheric asymmetry. Neurobiol. Aging 28, 272-283.

Fabiani, M., and Friedman, D. (1995). Changes in brain activity patterns in aging: the novelty oddball. Psychophysiology 32, 579-594.

Fabiani, M., Friedman, D., and Cheng, J. (1998). Individual differences in P3 scalp distribution in older adults, and their relationship to frontal lobe function. Psychophysiology 35, 698-708. Cambridge University Press.

Fabiani, M., Gratton, G., and Federmeier, K. (2007). "Event related brain potentials," in Handbook of Psychophysiology, 3rd Edn, eds J. Cacioppo, L. Tassinary, and G. Berntson (New York, NY: Cambridge University Press), 85-119.

Fabiani, M., and Gratton, G. (in press). "Aging, working memory and attention control: a tale of two processing streams?" in Principles of Frontal Lobe Function, 2nd Edn. Eds D. T. Stuss and R. T. Knight (New York, NY: Oxford University Press, Inc.).

Fabiani, M., Low, K. A., Wee, E., Sable, J. J., and Gratton, G. (2006). Reduced suppression or labile memory? Mechanisms of inefficient filtering of irrelevant information in older adults. J. Cogn. Neurosci. 18, 637-650.

Fjell,A.M., and Walhovd,K. B. (2001).P300 and neuropsychological tests as measures of aging: scalp topography and cognitive changes. Brain Topogr. 14, 25-40.

Friedman, D., Cycowicz, Y.M., and Gaeta, H. (2001). The novelty P3: an eventrelated brain potential (ERP) sign of the brain's evaluation of novelty. Neurosci. Biobehav. Rev. 25, 355-373.

Friedman, D., Kazmerski, V., and Fabiani, M. (1997). An overview of age-related changes in the scalp distribution of P3b. Electroencephalogr. Clin. Neurophysiol. 104, 498-513.

Gazzaley, A., Cooney, J. W., Rissman, J., and D'Esposito, M. (2005). Top-down suppression deficit underlies working memory impairment in normal aging. Nat. Neurosci. 8, 1298-1300.

Goldman-Rakic, P. S. (1987)."Circuitry of primate prefrontal cortex and regulation of behavior by representational memory," in Handbook of Physiology Sect. 1, Vol. 5, Pt. 1, eds V. Mountcastle and F.Plum (Bethesda,MD: American Physiological Society), 373-417.

Grady, C. L., McIntosh, A. R., Bookstein, F., Horwitz, B., Rapoport, S. I., and Haxby, J. V. (1998). Age-related changes in regional cerebral blood flow during working memory for faces. Neuroimage 8, 409-425.

Gratton, G., Coles, M. G., and Donchin, E. (1989). A procedure for using multi-electrode information in the analysis of components of the event-related potential: vector filter. Psychophysiology 26, 222-232.

Gratton, G., Coles, M. G. H., and Donchin, E. (1983). A new method for off line removal of ocular artifact. Electroencephalogr. Clin. Neurophysiol. $55,468-484$.

Gratton, G., Rykhlevskaia, E., Wee, E., Leaver, E., and Fabiani, M. (2009). Does white matter matter? Spatiotemporal dynamics of task switching in aging. J. Cogn. Neurosci. 21, 1380-1395.

Hambrick, D. Z., and Engle, R. W. (2002). Effects of domain knowledge, working memory capacity, and age on cognitive performance: an investigation of the knowledge-is-power hypothesis. $\operatorname{Cog} n$. Psychol. 44, 339-387.

Hasher, L., Lustig, C., and Zacks, R. (2008). "Inhibitory mechanisms and the control of attention," in Variation in Working Memory, eds A. R. A. Conway, C. Jarrold, M. J. Kane, A. Miyake, and J. N.Towse (New York: Oxford University Press), 227-249.

Hasher, L., and Zacks, R. T. (1988). "Working memory, comprehension, and aging: a review and a new view," in The Psychology of Learning and Motivation, ed. G. H. Bower (San Diego, CA: Academic Press), 193-225.

Heaton, R. K. (1981). Wisconsin Cards Sorting Test Manual. Odessa, FL: Psychological Assessment Resources.

Johnson, R. Jr. (1986). A triarchic mode of P300 amplitude. Psychophysiology 23, 367-384.

Jost, K., Mayr, U., and Rösler, F. (2008). Is task switching nothing but cue priming? Evidence from ERPs. Cogn. Affect. Behav. Neurosci. 8, 74-84.

Kane, M. J., and Engle, R. W. (2000) Working-memory capacity, proactive interference, and divided attention: limits on long-term memory retrieval. J. Exp. Psychol. Learn. Mem. Cogn. 26, 336-358.
Kendrick, D. C., and Watts, G. D. (1999). The Kendrick Assessment Scales of Cognitive Ageing. Windsor: NFER-Nelson.

Klein, M., Coles, M. G., and Donchin, E. (1984). People with absolute pitch process tones without producing a P300. Science 223, 1306-1309.

Knight, R. T. (1987). Aging decreases auditory event-related potentials to unexpected stimuli in humans. Neurobiol. Aging 8, 109-113.

Kray, J., Li, K. Z., and Lindenberger, U. (2002). Age differences in task switching components: the role of task uncertainty. Brain Cogn. 49, 363-381.

Kutas, M., McCarthy, G., and Donchin, E. (1977). Augmenting mental chronometry: The $\mathrm{P} 300$ as a measure of stimulus evaluation time. Science 197, 792-795.

Magliero, A., Bashore, T. R., Coles, M. G., and Donchin, E. (1984). On the dependence of P300 latency on stimulus evaluation processes. Psychophysiology 21, 171-186.

Mayeux, R., Stern, Y., Rosen, J., and Leventhal, J.(1981). Depression, intellectual impairment and Parkinson's disease. Neurology 31, 645-650.

McCarthy, G., and Donchin, E. (1981). A metric for thought: a comparison of P300 latency and reaction time. Science 211,77-80.

McCarthy, G., and Wood, C. C. (1985). Scalp distributions of event-related potentials: an ambiguity associated with analysis of variance models. Electroencephalogr. Clin. Neurophysiol. 62, 203-208.

Moscovitch, M., and Winocur, G. (1992) "The neuropsychology of memory and aging," in The Handbook of Aging and Cognition, eds F. I. M. Craik and T. A. Salthouse (Hillsdale, NJ: Erlbaum), 315-372.

Park, D. C., Polk, T. A., Mikels, J. A., Taylor, S. F., and Marshuetz, C. (2001). Cerebral aging: integration of brain and behavioral models of cognitive function. Dialogues Clin. Neurosci. 3 , 155-165.

Park, D. C., Smith, A. D., Lautenschlager, G., Earles, J., Frieske, D., Zwahr, M., and Gaines, C. (1996). Mediators of longterm memory performance across the life span. Psychol. Aging 11, 621-637.

Polich, J. (2007). Updating P300: an integrative theory of $\mathrm{P} 3 \mathrm{a}$ and $\mathrm{P} 3 \mathrm{~b}$. Clin. Neurophysiol. 118, 2128-2148.

Prabhakaran, V., Smith, J. A., Desmond, J. E., Glover, G. H., and Gabrieli, J. D. (1997). Neural substrates of fluid reasoning: an fMRI study of neocortical activation during performance of the Raven's progressive matrices test. Cogn. Psychol. 33, 43-63.

Rabbitt, P. (1965). An age decrement in the ability to ignore irrelevant information. J. Gerontol. 20, 233-238. 
Raven, J. C., Court, J. H., and Raven, J. (1977). Standard Progressive Matrices. London: H. K. Lewis.

Reuter-Lorenz, P. A., and Cappell, K. A. (2008). Neurocognitive aging and the compensation hypothesis. Curr. Dir. Psychol. Sci. 17, 177-182.

Rhodes, M. G., and Kelley, C. M. (2005). Executive processes, memory accuracy, and memory monitoring: an aging and individual differences analysis. J. Mem. Lang. 52, 578-594.

Rösler, F. (1983). "Endogenous ERPs and cognition: probes, prospects, and pitfalls in matching pieces of the mind-body problem," in Tutorials in Event-Related Potential Research: Endogenous Components, eds A. W. K. Gaillard, and W. Ritter (Amsterdam: Elsevier), 9-35.

Salthouse, T. (1992). Mechanisms of AgeCognition Relations in Adulthood. New Jersey: Lawrence.

Schneider-Garces, N. J., Gordon, B. A., Brumback-Peltz, C. R., Shin, E., Lee,
Y., Sutton, B. P., Maclin, E. L. Gratton, G., and Fabiani, M. (2010). Span, CRUNCH and beyond: working memory capacity and the aging brain. J. Cogn. Neurosci. 22, 655-669.

Sokolov, E. N. (1963). Higher nervous functions: the orienting reflex. Annu. Rev. Physiol. 25, 545-580.

Spieler, D. H., Balota, D. A., and Faust, M. E,(1996). Stroop performance in healthy younger and older adults and in individuals with dementia of the Alzheimer's type. Exp. Psychol. Hum. Percept. Perform. 22, 461-479.

Squires, K., Petuchowski, S., Wickens, C., and Donchin, E. (1977). The effects of stimulus sequence on event related potentials: a comparison of visual and auditory sequences. Percept. Psychophys. 22, 31-40.

Squires, K. C., Wickens, C., Squires, N. K., and Donchin, E. (1976). The effect of stimulus sequence on the waveform of the cortical event-related potential. Science 193, 1142-1146.
Sunghan, K., Hasher, L., and Zacks, R. T. (2007). Aging and a benefit of distractibility. Psychon. Bull. Rev. 14, 301-305.

Sutton, S., Braren, M., Zubin, J., and John, E. R. (1965). Evoked-potential correlates of stimulus uncertainty. Science 150, 1187-1188.

Sutton, S., Tueting, P., Zubin, J., and John, E. R. (1967). Information delivery and the sensory evoked potentials. Science 155, 1436-1439.

Urbach, T. P., and Kutas, M. (2002).The intractability of scaling scalp distributions to infer neuroelectric sources. Psychophysiology 39, 791-808.

Verhaeghen, P., and Cerella, J. (2002). Aging, executive control, and attention: a review of meta-analyses. Neurosci. Biobehav. Rev. 26, 849-857.

Verleger, R. (1988). Event-related potentials and memory: a critique of the context updating hypothesis and an alternative interpretation of P3. Behav. Brain Sci. 11, 343-356.
Conflict of Interest Statement: The authors declare that the research was conducted in the absence of any commercial or financial relationships that could be construed as a potential conflict of interest.

Received: 30 April 2011; accepted: 26 July 2011; published online: 18 August 2011.

Citation: Peltz CB, Gratton G and Fabiani $M$ (2011) Age-related changes in electrophysiological and neuropsychological indices of working memory, attention control, and cognitive flexibility. Front. Psychology 2:190. doi: 10.3389/fpsyg.2011.00190

This article was submitted to Frontiers in Cognition, a specialty of Frontiers in Psychology.

Copyright (c) 2011 Peltz, Gratton and Fabiani. This is an open-access article subject to a non-exclusive license between the authors and Frontiers Media SA, which permits use, distribution and reproduction in other forums, provided the original authors and source are credited and other Frontiers conditions are complied with. 